\title{
Penis evolution across species: divergence and diversity
}

\author{
David J. Hosken ${ }^{1}$, C. Ruth Archer ${ }^{1}$, Clarissa M. House ${ }^{2}$ \& Nina Wedell ${ }^{1}$ \\ ${ }^{1}$ Centre for Ecology \& Conservation, University of Exeter, Cornwall, Penryn TR109EZ, UK \\ ${ }^{2}$ School of Science and Health \& Hawkesbury Institute for the Environment, Western \\ Sydney University, Hawesbury, Richmond NSW 2753, Australia
}

Correspondence to: D.J.H.d.j.hosken@exeter.ac.uk

\begin{abstract}
The penis is an incredibly diverse and rapidly evolving structure, such that even in closely related species that otherwise differ very little in their morphology, penis form can be highly differentiated. Penises are also much more complex than their fundamental function - sperm transfer - would seem to require. The rapid divergent evolution of male structures is typically the signature of traits under sexual selection and the current evidence suggests the penis is no different in this regard. Despite the general agreement that sexual selection is the main driver of penis evolution, many questions about penis evolution remain unresolved. Furthermore, the penis might be an ideal characteristic on which to focus in the drive to link phenotype with genotype.
\end{abstract}

\section{[H1]Introduction}

The penis of internally fertilizing species is a structure that facilitates delivery of sperm and their DNA payload from one organism to another. This transfer is fundamental for most sexual reproduction. However, despite this seemingly simple brief, the variation in penis form (and other, closely associated, genital structures: herein all referred to as the penis) across species is extraordinary, so much so that even penis number can vary interspecifically (Fig. 1) ${ }^{1}$. Thus, penis length can exceed body length ${ }^{2}$, some earwigs have two penises ${ }^{3}$, many marsupials and snakes have a bifurcate penis with two sets of glans ${ }^{1}$, and - in rare cases - the female possesses the intromittent organ that is inserted into the male rather than the other way around ${ }^{4}$.

Penis variation has been central to taxonomy, because even in closely related species that otherwise vary very little, penis form can be highly differentiated ${ }^{1,5}$ (Fig 1). This general pattern is observed across all animals with internal fertilization ${ }^{1}$ and indicates 
that the penis evolves very rapidly and divergently. Until about the 1990s the reasons for this pervasive pattern were not clear6, but the current balance of evidence suggests that sexual selection is the principal driver of penis evolution ${ }^{1,7,8}$. Indeed, rapid divergent evolution of male characteristics is the hallmark of sexual selection ${ }^{1,11}$. For example, deer antlers used in male-male competition for females have similarly evolved rapidly and divergently, so that marsh deer (Blastocerus dichotomus) have simple forked antlers, whereas their close relative the moose (Alces alces) have large palmate antlers ${ }^{9}$. Similar patterns are also evident in dung beetle horns ${ }^{10}$ and in the male colour-patterns favoured by female mate-choice in waterfowl ${ }^{11}$. In this Review, we will consider the evidence for sexual selection as the major driver of penis evolution, while also discussing situations in which natural selection acts on male genitals and currently unresolved issues in the field.

\section{[H1] Explanations for the diversity}

Historically, several explanations for the broad pattern of rapid divergent evolution had been proposed 1,7,8. Some of these hypotheses involved sexual selection, others invoke natural selection, and some even suggest that genital evolution was selectively neutral ${ }^{1,7,8}$. The ecological hypothesis suggests that natural selection acts on penis form independently of mating ${ }^{12}$. This situation would occur when genital growth requires high resource investment and so genitals use resources that could otherwise be allocated towards survival for example. This is essentially what occurs in the beetle Onthophagus nigriventris where trade-offs occur between investment in weapons (horns) and investment in testes ${ }^{13}$. Alternatively, natural selection might directly act on the penis if it is particularly conspicuous and so might attract the attention of predators or, if it reduces how well individuals move, making it difficult for them to escape predators or catch prey as can occur with some fish ${ }^{12}$. A variant on this idea is that ecology can interact with sexual selection to affect genital form, for example, the presence of predators might select on genital morphology to promote speedier sperm transfer, reducing the time that more vulnerable mating fish could be attacked for example ${ }^{14}$. Good evidence for the ecology hypothesis exists in some taxa, particularly fish ${ }^{12,14}$, but the general applicability of this idea is unclear, if only because, for many taxa, the penis is hidden when not in use ${ }^{1}$.

The lock and key hypothesis suggests that species have very specific genital form to prevent hybridization. That is, successful mating can only occur if there is a close match 
between the female reproductive tract (the lock) and the penis (the key). This hypothesis involves a naturally selected rather than a sexually selected benefit, with hybrids selected against, as they have neither the right lock nor key ${ }^{1,7,8}$. However, this idea is not supported by within and between species geographical variation in genitalia, within-species variation in genital form, the morphology of female reproductive tracts, or the associations between male and female genitals during copulation 1,7 (but see e.g. $15,16)$.

The pleiotropy hypothesis suggests that the penis evolves via the effects of selection on other traits and that penis form itself is selectively neutral ${ }^{1,7,8}$. The hypothesis requires genes to affect more than one trait (that is, they have pleiotropic effects) and that selection on characteristics genetically linked to genital form can shape penis evolution. Some evidence does suggest that genitals are subjected to pleiotropic gene effects, with penis length correlated with leg length in a waterstrider (Gerris incognitus) for example 6 . However, why genitals should be affected by pleiotropy any more (or less) than other traits is not clear ${ }^{1,7}$.

\section{[H1] Sexual selection \& evolution}

Before considering the role of sexual selection in penis evolution, what is meant by sexual selection and what conditions are needed for this selection to result in evolution must be clarified. Sexual selection can be thought of as reproductive competition between members of the same sex and species $11,17,18$. If some characteristics of an individual increase the likelihood of mating and/or securing fertilizations - and as a result these individuals produce more offspring - the characteristics providing the advantage will be under sexual selection. For example, in many species males with brighter colours attract more mates, and therefore, male colour is sexually selected ${ }^{11}$. Two general mechanisms of sexual selection generate reproductive advantage - mate choice and mate competition, and typically females do most choosing and males do most competing ${ }^{11}$.

For sexual selection to result in the evolution of the characteristics generating the reproductive advantage, genetic variation for said characteristics must be present: that is, these traits have to be heritable; offspring must resemble their parents. All these requirements are captured very simply in the univariate breeders' equation

$$
r=s h^{2}
$$


where $r$ is the response to selection (that is, evolution), $s$ is the strength of selection (the difference between the population mean and the breeders' mean for the trait), and $h^{2}$ is the trait heritability (a measure of how much offspring are expected to resemble their parents because of shared genes). As is clear from the equation, if there is no selection or genetic variation (either $s$ or $h^{2}=0$ ), no evolution can occur (anything multiplied by 0 $=0$ ). Crucially, this simple equation shows that selection and evolution are not the same thing $(r \neq s)$.

\section{[H1] Postcopulatory selection}

In the context of penis evolution (and for sake of linguistic simplicity, assuming the general case of males having a penis), the penis will evolve if some of its characteristics influence siring success (selection) and if sons resemble their fathers in that attribute (heritability). Sperm competition and cryptic female choice, the typical mechanisms of sexual selection that operate after coupling (Fig. 2), are expected to be especially important in generating selection on the penis because male genitals are often concealed before copulation begins and cannot, therefore, always influence whether or not mating occurs. These two mechanisms of sexual selection that operate during and after mating (or egg and/or sperm release in external fertilizers), are, therefore, thought to be especially important for penis evolution ${ }^{1,7,8}$.

Sperm competition occurs when the sperm from two or males compete to fertilize a female's ova ${ }^{19}$. In internal fertilizers, that is when females store sperm from more than one male or matings occur in quick succession so that ejaculates from multiple males are present in the female reproductive tract ${ }^{19}$. Sperm competition is likely to have been particularly important in the origin of the penis, as the existence of an intromittent organ would enable males to place more sperm closer to the ova and, therefore, outcompete their rivals ${ }^{19}$.

Cryptic female choice (CFC) occurs when any female phenotype (behavioural, morphological, or physiological) alters male fertilization probability based on some element of the male phenotype ${ }^{20,21}$. CFC includes, but is not restricted to, biased sperm usage (i.e. using the sperm from some males more than others) and can also include selective sperm storage for example 20,21 . This has been demonstrated in chickens where females eject sperm after mating with subordinate males ${ }^{22}$. Both CFC and sperm competition can act on male traits beyond just genitalia, with sperm form, semen 
composition and copulatory behaviour just some characters also subjected to these mechanisms of selection 19,21,23. Furthermore, male stimulation of females during copulation (copulatory courtship) is widespread ${ }^{24}$ and this can influence siring success ${ }^{25}$, while in humans there have been suggestions that a male's ability to stimulate a female and induce orgasm during copulation could affect sperm retention $26-28$, but the current evidence is equivocal28. As an aside, it should be noted that arguments about the adaptive nature (or not) of human female orgasm will not be resolved by simple measurement of clitoral size ${ }^{29}$.

Operationally separating the two postcopulatory mechanisms is difficult, as ejaculates compete within the female in internal fertilizers, so in a way females define the rules of engagement ${ }^{20,21}$. Additionally, testis size indicates that female multiple mating and sperm competition are common (Box 1), even in humans (Fig. 4)20,21,28,30,31 so in some sense CFC might always be operating20,21. However, with clever experimental approaches, variation in paternity can be partitioned between male and female effects. For example, using standardized male and female genotypes should enable variation in paternity to be attributable to males or females. This has been done using Drosophila genotypes to show distinct male and female (and interaction) effects ${ }^{32}$ and work with guppies has used artificial insemination to show that post-copulatory sexual selection reinforces pre-copulatory affects - colourful males are always selectively favoured ${ }^{33}$. A combination of these approaches would be revealing. It should also be noted that genital form can affect mating success in some instances ${ }^{34,35}$. That is, there can be precopulatory selection on genitals as documented in a flour beetle (Gnatocerus cornutus) where males with an intermediate sized penis obtained more matings ${ }^{35}$.

\section{[H1]Sexual selection on the penis}

The evidence that sexual selection is the primary agent acting on penis form is now incontrovertible and comes in the form of comparative analyses, experimental evolution studies, and directly estimating selection in extant populations ${ }^{1,7,8}$. Studies have also tested the genetic assumption that penis form is heritable (see below).

The most important comparative study of penis evolution compared the complexity of penis form in insect groups that were either monogamous and, therefore, do not experience postcopulatory sexual selection, or polyandrous, whereby females mate with multiple males and postcopulatory sexual selection occurs ${ }^{36}$. If sexual selection was 
important in driving penis evolution, then genitals in the polyandrous insects should be more differentiated than in the monogamous group. Indeed, use of geometric morphometrics to describe penis shape variation demonstrated that genitals of groups with postcopulatory sexual selection (polyandrous) had evolved far more quickly and divergently than in those without sexual selection (monogamous) (Fig. 3) ${ }^{36}$. Similarly, across groups of mammals, penis form is correlated with testis size ${ }^{37}$, which is a measure of the strength of postcopulatory sexual selection ${ }^{38-42}$ (Box 1). Finally if sexual selection was acting on penis form, then one would predict patterns of coevolution between male and female genital form, and, indeed this pattern is generally observed 43 . However, male-female coevolution is not exclusively supportive of penis evolution through sexual selection, because natural selection to prevent hybridization (the lock and key hypothesis), which will also generate correlated evolution in male and female genital form ${ }^{8}$ - although this distinction (natural vs. sexual selection) depends the precise mechanism selecting against hybirds ${ }^{8}$.

In addition to comparisons across species, many studies have now documented the covariance between penis form and mating or fertilization success within populations, measuring selection currently acting on the penis 7,8 . Such studies include work on beetles, waterstriders, damselflies, moths, and mice, all of which show that penis form influences male reproductive success ${ }^{35,44-48}$ (Fig. 2). Evidence also suggests sexual selection on human penis size ${ }^{49,50}$ (discussed below), and female mosquito fish tend to prefer to associate with larger males that have a longer penis ${ }^{51}$. Interestingly, if formal estimates of selection on genital form are compared with those on general morphology, no significant differences between the two are evident (Table 1). That is, despite the more rapid evolution of male genitals compared with general morphology (Fig. 1), selection does not seem to be especially strong on penis form (remember responses to selection will depend in part on the strength of selection: $r=s h^{2}$ ). If this finding is generally true, it suggests that, despite evolving rapidly, contemporary selection on genital form is not always especially strong (note however sample sizes in Table 1 are small).

For selection to result in evolution, a minimum requirement is that penis form is heritable. In addition to the many studies directly documenting selection on the penis $25,35,44-48$ a large number of studies have now documented substantial heritable variation in genital form $2,6,52-56$ with estimates from these studies suggesting that 10 $50 \%$ of the variation in genital form is due to genetic variation ${ }^{2}$ (strictly speaking 
additive genetic variation: the rest is generated by the environment and gene-gene or gene-environment interactions for example). Interestingly, in some species, genital form is relatively insulated from nutritional stress ${ }^{35,57}$, which suggests that the penis is reasonably developmentally canalized (that is, the penis is relatively resistant to developmental insult) ${ }^{35,57}$. Furthermore, this inference (canalization) is consistent with patterns of penis scaling relative to body size: genital size tends to be negatively allometric, which means that as body size increases (decreases), penis size increases (decreases) at a lesser rate ${ }^{58,59}$. Thus larger males tend to have a relatively smaller penis 58,59 .

Male genitals have also been studied using experimental evolution. This involves establishing replicate populations, which are then experimentally forced to evolve with and without sexual selection by allowing mate choice and mate competition (housing animals together) or by experimentally enforcing monogamy (housing animals in pairs). These studies indicate that sexual selection affects penis evolution in flies, beetles, and mice (Fig. 3) ${ }^{53-55 .}$

\section{[H2] Sexual conflict: the damaging penis}

Reproduction is often viewed as a cooperative affair, but, in fact, intense evolutionary conflict exists between the sexes over reproductive decisions ${ }^{60,61}$. Although sexual conflict can be thought of as an evolutionary struggle in which both sexes try to gain the upper hand to enhance their own fitness, the conflict can be latent and not generate selection. That is, even though sexual conflict will always be present in nature, it will not always generate selection ${ }^{60-63}$. For example, a male's fitness could be enhanced if females mated more, but if males cannot coerce (or otherwise manipulate) females into additional mating, then a conflict over mating frequency exists, but it does not generate any additional selection 60,61 . Sexual conflict can, therefore, be thought of as potential for selection in much the same way that variance in fitness is potential for selection ${ }^{60}$. When conflict does generate selection, unresolvable co-evolutionary arms races can occasionally ensue ${ }^{61,63}$. That is, females and males can become trapped in endless cycles of adaptation and counter-adaptation that cannot be resolved. Deciding whether sexual conflict exists over a specific trait is conceptually easy, by simply asking what would happen to trait values if they were totally controlled by one sex or the other64. If values would change under single-sex control, conflict exists over the trait. Importantly, traits that have evolved to provide competitive reproductive advantages over same-sex rivals can also generate male-female conflict, as in the case of the spiny genitalia of some 
beetles, which enhance sperm competitiveness but damage females65,66. Thus, sexual conflict can also generate sexual selection, and traits that are harmful to one sex can spread through populations as long as the advantage to the other sex outweighs the cost of harm 60,61 .

As noted conflict can extend to penis morphology as males attempt to manipulate females to male advantage. For example, dungflies (Sepsis cynipsea) and seed-beetles (Callosobruchus maculatus) have a penis that is covered with spikes and spines that damage females during mating; scars are observed within the female reproductive tract after copulation ${ }^{65-67}$. Damaging females this way might reduce the likelihood that they remate with rival males, or might enable access of seminal fluid to the female nervous system to manipulate females for male benefit - increasing their egg laying for example ${ }^{60}$. In seed-beetles, damage to females seems to be the result of sperm competition, as males with more damaging penises are superior sperm competitors they sire more offspring66. This provides an excellent example of male-male competition being harmful to females ${ }^{66,68}$. Interestingly, female beetles have evolved in response to this harm, as in species where males have longer penis spines females have thicker vaginal endometria, which limits the likelihood that males will penetrate the vaginal wall 69 .

Traumatic insemination, whereby the penis (or its equivalent) does not enter the vagina but is instead used to pierce the female body wall, is perhaps the ultimate example of penetration harming the female68. This process has been best characterized in bedbugs, in which the penis is used to stab the female and insemination occurs directly into the body cavity close to the ovaries ${ }^{70}$. This tactic has presumably evolved as a male means of hijacking female reproductive interests by circumventing the normal route of insemination which provides females some control over who fertilizes their ova ${ }^{70}$. Interestingly, in many species of bedbug, females have evolved an external groove to guide the penis into what is essentially a secondary reproductive tract ${ }^{70,71}$. Evolution of this groove and associated structures probably represents females reducing the costs of being stabbed and reasserts their control over fertilization ${ }^{70}$. Interestingly, in species with traumatic insemination, the stabbing device seems to violate the general rule of rapid divergent evolution of intromittent organs, as demonstrated by the fact that bedbugs and spiders have all converged on similar designs that essentially resemble hypodermic needles 68,72 . 
[H2]Is it all sexual selection?

Despite the general agreement that sexual selection is the primary driver of male genital evolution, evidence suggests that natural selection can also affect genital form $54,73,74$. At present, this is largely restricted to a few species and studies (see below), but good evidence is now available from some fish species (members of the Poeciliidae) to suggest that penis size is affected by predation ${ }^{73,74}$. In populations with high levels of predation, males tend to have smaller intromittent organs despite the fact that females prefer males with a larger penis 73,74 . This situation seems to arise because a larger penis slows male swimming speed, so males with a larger penis are more likely to be eaten when lots of predators are present ${ }^{74}$. Experimental evolution in the fly Drosophila simulans also indicates that evolving with elevated natural selection - in this case, exposure to high temperature - can affect genital form, although it is not clear why ${ }^{54}$. Across snail species, penis form tends to be less similar when species ranges overlap geographically ${ }^{15}$, which is consistent with natural selection against hybridization ${ }^{1}$. Thus, although sexual selection is largely responsible for penis evolution, natural selection can at least sometimes also affect penis form.

\section{[H1] Evolution of the human penis}

Much of the data regarding penile evolution comes from non-humans, raising the question of how much research in other taxa can reveal about the evolution of the human penis. Given the general congruence of the across-species evidence, Occam's razor suggests the human penis will have been subject to sexual selection like the intromittent organs of other species, causing the same rapid evolution seen in other taxa. Furthermore, before humans began wearing clothes, the penis would have been visible to potential mates (and competitors), and so could have influenced mating opportunities, especially considering recent evidence indicating penis size influences female choice ${ }^{49,50 .}$ One study investigating female assessment of digital projections of life-size, computergenerated images of men with a flaccid penis, revealed that women preferred larger penises, particularly in tall men, but that this preference decelerated once penis length exceeded about $7.6 \mathrm{~cm}^{49}$. That is, very large penises were not as preferred, which may reflect how our nervous systems assess stimuli differences. Weber's law of cognitive bias in signal processing shows that a unit increase in a signal (like penis size may have been) will have less effect on the receiver (females) as the signal get progressively larger ${ }^{75}$, which could explain the decreased preference strength as penis size increased. Moreover, both increased penis size and height had equally positive effects on how 
women rated model attractiveness, although the hip:shoulder width ratio of models had the greatest effect ${ }^{49}$. Other work has also revealed that preference varied depending on whether women were assessing men for brief encounters or long-term relationships ${ }^{50}$, a finding reflected in many in studies of human sexuality - sexual behaviours often vary depending on context ${ }^{28}$. Notably, although this work suggests that overt female choice could act on male penis form, these studies used statements of preference rather than actual measures of male fitness. That is, it is not clear that these reported preferences would result in selection. Additionally, it is not clear if flaccid penis size correlates with erect size. In fact the evidence suggests that unless the flaccid penis is stretched, the correlation between erect and flaccid length is at best very weak ${ }^{76}$.

So the direct evidence for sexual selection on the human penis is equivocal, but humans have a relatively large penis compared with most of our closest living relatives, the great $\operatorname{apes}^{30}$ (Fig 4). Additionally, testis size in humans is consistent with the expected levels of sperm competition our species underwent during its evolutionary divergence from our great ape ancestors 28,31 , hinting that penis form could also be subject to selection after intromission. Interestingly, unlike the great apes, we lack a penis bone (baculum) ${ }^{30}$. At present the precise function of the baculum in primates is not clear ${ }^{30}$ although there is evidence across mammals that it is sexually selected 37,55 . It is interesting to speculate why humans lost the baculum. It is even possible to conjure multiple adaptive explanations for its loss. But prosaically, perhaps our small population sizes (from a genetic not census perspective, and certainly historically) and the heightened probability of stochastic events (genetic drift) in small populations are all we need to invoke - stochastic events may mean the character was lost.

\section{[H1]Known unknowns}

Evidence suggests that male genital form is relatively developmentally buffered within species, for good reason - the penis has to fit most females and, therefore, evolution should favour relative invariable form intraspecifically - this is not always true and some fly species show considerable plasticity in genital form ${ }^{77}$. At present it is not clear when developmental canalization is to be expected and when not. However, if female genital form (or preference for male genital form) is highly variable across environments, then the same should be true of the penis. 
Additionally, in many instances the precise physiological or neurological mechanisms through which the penis generates differential fertilization or mating success are not clear. One well-studied exception to this generality is in a damselfly (Calopteryx haemorrhoidalis). Here the penis stimulates mechanoreceptors (designed to detect egg movement) in the female reproductive tract to cause the sperm from rival males to be ejected from the female sperm stores ${ }^{45,78}$. However, in general, the proximate female mechanisms that generate selection on penis form are not well defined, and this area would benefit from much more work, especially given the plethora of cryptic female choice mechanisms (differential sperm storage and usage for example) that could act on genital form ${ }^{20}$.

Potential damage done to females by the penis during copulation is also somewhat enigmatic ${ }^{79,80}$. In at least some cases, this damage greatly shortens female life expectancy and fitness, and seemingly any male able to reduce female damage while retaining male benefits would be at a selective advantage - their mates would continue to produce their offspring instead of dying. Thus, although we have a good theoretical understanding of male harm ${ }^{60}$, empirical evidence lags behind theory to an extent. We have also not explored the possibility of females damaging male genitalia and potential benefits that this could bring.

Finally, despite rapid divergence, selection on genitals may not be particularly strong (Table 1). Obviously this conclusion is drawn with all the caveats that apply to estimating selection in general (estimates are imprecise and are time and place specific) and our comparison (Table 1) is fairly simplistic. However, if selection is not particularly strong - as the data superficially imply - then one possible explanation for rapid evolution is that some feature of the genetic architecture of the penis facilitates it. For example, genitals may not be genetically integrated with rest of phenotype and are therefore free to evolve without being constrained by selection on other characters. That is, perhaps pleiotropic (or linkage) effects do not retard genital evolution to the extent they affect other traits and this may be especially true because the penis enjoys sex-limited development. All this needs further work.

\section{[H1] Future possibilities}

One of the holy grails of modern biology is to link phenotype with genotype and establishing this link offers the potential to realize the full promise of the 'omics' era. However, genotypes and phenotypes are very complicated and to begin to link the two, 
perhaps a focus on traits with simple, well-defined function is needed. Given the relatively simply function of the penis, to deliver sperm and stimulate the female, penis size and shape could be an excellent trait on which to focus to make the link, despite the huge variation in penis form. This could be particularly fruitful if genitals are not genetically integrated (which they may not be: see above), because this means there really will be "genes for genitals". That is, the genes affecting genitals will not affect other characters, and hence finding them and establishing what affects they have could be relatively simple.

\section{[H1]Conclusions}

Penis phenotypes are well described for many species, owing, in part, to their contribution to taxonomy and the many functional studies undertaken by evolutionary biologists, which include statistical-genetic characterisations and QTL identification. Perhaps now is the time to apply genomic approaches and generate the data needed to fully understand this extraordinarily variable organ.

\section{Acknowledgements}

We thank Michelle Taylor for the Table 1 analysis and many colleagues for discussion of genital evolution.

\section{Referee accreditation}

Nature Reviews Urology thanks XX. XX, and other unnamed reviewers for their help with the peer review of this manuscript.

1. Eberhard, W. G. Sexual Selection and Animal Genitalia. (Harvard University Press, London, 1985).

2. Higgins, S., Hosken, D.J., \& Wedell, N. Phenotypic and genetic variation in male genitalia in the seedbug, Lygaeus equestris (Heteroptera). Biol. J. Linn. Soc. 98, 400-405 (2009).

3. Kamimura, Y. \& Matsuo, Y. A "spare" compensates for the risk of destruction of the elongated penis of earwigs (Insecta: Dermaptera). Naturwiss. 88, 468-471 (2001) 
4. Yoshizawa, K., Ferreira, R. L., Kamimura, Y., \& Leinhard, C. Female penis, male vagina, and their correlated evolution in a cave insect. Curr. Biol. 24, 1006-1010 (2014).

5. Rowe, L. \& Arnqvist, G. Sexual selection and the evolution of genital shape and complexity in water striders. Evolution 66, 40-54 (2011).

6. Arnqvist, G. \& Thornhill, R. Evolution of animal genitalia: patterns of phenotypic and genotypic variation and condition dependence of genital and non-genital morphology in waterstriders (Heteroptera: Gerridae: Insecta). Gen. Res. 71, 192-212 (1998).

7. Hosken, D. J. \& Stockley, P. Sexual selection and genital evolution. Trends Ecol. Evol. 19, 87- 93 (2004).

8. Simmons, L. W. Sexual selection and genital evolution. Austr. Entomol. 53, 1-17 (2014).

9. Pitra, C., Fickel, J., Meijaard, E., \& Groves, P. C. Evolution and phylogeny of old world deer. Mol. Phyl. Evol. 33, 880-895 (2004)

10. Emlen, D. J., Marangelo, J., Ball, B. \& Cunningham, C. W. Diversity in the weapons of sexual selection: horn evolution in the beetle genus Onthophagus (Coleoptera: Scarabaeidae). Evolution 59, 1060-1084 (2005).

11. Andersson, M. Sexual Selection. (Princeton University Press, Princeton, 1994).

12. Langerhans, R. B. in Ecology and Evolution of Poeciliid Fishes (eds Evans, J., Pilastro, A. \& Schlupp, I.) 228-240 (University of Chicago Press, Chicago, 2011).

13. Simmons, L. W. \& Emlen, D. J. Evolutonary trade-off between weapons and testes. Proc. Natl. Acad. Sci. USA 103, 16346-16351 (2006).

14. Heinen-Kay, J. L. \& Langerhans, R. B. Predation-associated divergence of male genital morphology in a livebearing fish. J. Evol. Biol. 26, 2135-2146 (2013).

15. Hollander, J., Smadja, C. M., Butlin, R. K. \& Reid, D. G. Genital divergence in sympatric sister snails. J. Evol. Biol. 26, 210-215 (2013).

16. Kameda, Y., Kawakita, A. \& Kato, M. Reproductive character displacement in genital morphology in Satsuma land snails. Am. Nat. 173, 689-697 (2009).

17. Darwin, C. The Descent of Man and Selection in Relation to Sex. (John Murry, London, 1871).

18. Hosken, D. J. \& House, C. M. Sexual selection. Curr. Biol. 21: R62-R65 (2011).

19. Parker, G. A. in Sperm Competition and the Evolution of Animal Mating Systems (ed. Smith, R. L.) 1-60 (Academic Press, London, 1984).

20. Eberhard, W. G. Female Control: Sexual Selection by Cryptic Female Choice (Princeton University Press, Princeton, 1996). 
21. Pitnick, S. \& Hosken, D. J. in Evolutionary Behavioural Ecology (eds. Westneat, D. F. \& Fox, C. W.) 379-399 (Oxford University Press, Oxford, 2010).

22. Pizarri, T. \& Birkhead, T. R. Female feral fowl eject sperm of subdominant males. Nature 405, 787-789 (2000).

23. Hodgson, D. J. \& Hosken, D. J. Sperm competition promotes the exploitation of rival ejaculates. J. Theoret. Biol. 243, 230-234 (2006).

24. Eberhard, W. G. Evidence for widespread courtship during copulation in 131 species of insects and spiders, and implications for cryptic female choice. Evolution 48, 711-733 (1994).

25. Edvardsson, M. \& Arnqvist, G. Copulatory courtship and cryptic female choice in red flour beetles. Proc. R. Soc. Lond. B 267, 559-563 (2000).

26. Zervomanolakis, I., Ott, H. W., Hadziomerovic, D., Mattle, V., Seeber, B. E., Virgolini, I., et al. Physiology of upward transport in the human female genital tract. Reproductive Biomechanics 1101, 1-20 (2007).

27. Reeder, D. M. In Sexual Selection and Reproductive Competition in Primates: New Perspectives and Directions (ed. Jones C. B.) 255-303. (American Society of Primotologists, Norman, 2003).

28. Leivers, S. \& Simmons, L. W. Human sperm competition: playing a defensive strategy. Adv. Stud. Behav. 46, 1-44 (2014).

29. Hosken, D. J. Clitoral variation says nothing about female orgasm. Evol. Dev. 10, 393-395 (2008).

30. Dixson, A. F. Primate Sexuality. (Oxford University Press, Oxford, 1998).

31. Simmons, L. W., Firman, R. C., Rhodes, G. \& Peters, M. Human sperm competition: testis size, sperm production and rates of extra-pair copulations. Anim. Behav. 68, 297-302 (2004).

32. Clark, A. G., Begun, D. J. \& Prout, T. Female x male interactions in Drosophila sperm competition. Science 283, 217-220 (1999).

33. Evans, J. P., Zane, L. Francescato, S. \& Pilastro, A. Directional postcopulatory sexual selection revealed by artificial insemination. Nature 421, 360-363 (2003).

34. Fairbairn, D. J. in Sex, Size \& Gender: Evolutionary Studies of Sexual Size Dimorphism (eds. Fairbairn, D. J., Blanckenhorn, W. U. \& Szekely, T.) 97-105 (Oxford University Press, Oxford, 2009).

35. House, C. M., Sharma, M. D., Okada, K. \& Hosken, D. J. Pre and post-copulatory selection favor similar genital phenotypes in the male broad horned beetle. Integr. Comp. Biol. 56, 682-693 (2016). 
36. Arnqvist, G. Comparative evidence for the evolution of genitalia by sexual selection. Nature 393, 784-786 (1998).

37. Ramm, S. A. Sexual selection and genital evolution in mammals: a phylogenetic analysis of baculum length. Am. Nat. 169, 360-369 (2007).

38. Simmons, L. W. Sperm Competition and it Evolutionary Consequences in the Insects (Princeton University Press, Princeton, 2001).

39. Gage, M. J. G. Associations between body size, mating pattern, testis size and sperm length across butterflies. Proc. R. Soc. Lond. B. 258, 247-254 (1994).

40. Stockley, P., Gage, M. J. G., Parker, G. A. \& Moller, A. P. Sperm competition in fishes: the evolution of testis size and ejaculate characteristics. Am. Nat. 149, 933-954 (1997)

41. Hosken, D. J. Sperm competition in bats. Proc. R. Soc. Lond. B. 264, 385-392 (1997).

42. Hosken, D. J. \& Ward, P. I. Experimental evidence for testis size evolution via sperm competition. Ecol. Lett. 4, 10-13 (2001).

43. Brennan, P. L. R. et al. Coevolution of male and female genital morphology in waterfowl. PLoS One 5, e418 (2007).

44. Arnqvist, G. \& Danielsson, I. Copulatory behaviour, genital morphology, and male fertilization success in water striders. Evolution 53,147-156 (1999).

45. Córdoba-Aguilar, A. Male copulatory sensory stimulation induces female ejection of rival sperm in a damselfly. Proc. R. Soc. Lond., B. 266, 779-784 (1999)

46. House, C. M. \& Simmons, L. W. Genital morphology and fertilisation success in the dung beetle Onthophagus taurus: an example of sexually selected male genitalia. Proc. R. Soc. Lond. B. 270, 447- 455 (2003).

47. $\mathrm{Xu}, \mathrm{J} . \&$ Wang, Q. Form and nature of precopulatory sexual selection in both sexes of a moth. Naturwiss. 97, 617-625 (2010).

48. Stockley, P. et al. Baculum morphology predicts reproductive success of male house mice under sexual selection. BMC Biol. 11, 66 (2013).

49. Mautz, B. S., Wong, B. B. M., Peters, R. A. \& Jennions, M. D. Penis size interacts with body shape and height to influence male attractiveness. Proc. Natl. Acad. Sci. USA 110, 6925-6930 (2013).

50. Prause, N., Park, J., Leung, S. \& Miller, G. Women's preference for penis size: a new research method using selection among 3D models. PLoS One 10, e0133079 (2015). 
51. Kahn, A. T., Mautz, B. \& Jennions, M. D. Females prefer to associate with male with longer intromittent organs in mosquitofish. Biol. Lett. 6, 55-58 (2009).

52. House, C. M. \& Simmons, L. W. The evolution of male genitalia: patterns of genetic variation and covariation in the genital sclerites of the dung beetle Onthophagus taurus. J. Evol. Biol. 18, 1281-1292 (2005).

53. Simmons, L.W., House, C.M., Hunt, J. \& García-González, F. Evolutionary response to sexual selection in male genital morphology. Curr. Biol. 19, 14421446 (2009).

54. House, C.M., et al. Sexual and natural selection both influence male genital evolution. PLoS One 8, e63807 (2013).

55. Simmons, L. W. \& Firman, R. C. Experimental evidence for the evolution of the mammalian baculum by sexual selection. Evolution 68, 276-283 (2014).

56. Preziosi , R. F. \& Roff, D. A. Evidence of genetic isolation between sexually monomorphic and sexually dimorphic traits in the water strider Aquarius remigis. Heredity 8, 92-99 (1998).

57. House, C. M. \& Simmons, L. W. No evidence for condition-dependent expression of male genitalia in the dung beetle Onthophagus taurus. J. Evol. Biol. 20,13221332 (2007).

58. Hosken, D. J., Minder, A. M. \& Ward, P. I. Male genital allometry in Scathophagidae (Diptera). Evol. Ecol. 19, 501-515 (2005).

59. Eberhard, W. G., et al. One size fits all? Relationships between the size and degree of variation in genitalia and other body parts in twenty species of insects and spiders. Evolution 52, 415-431 (1998).

60. Lessells, C. M. Why are males bad for females? Models for the evolution of damaging male mating behaviour. Am. Nat. 165, S46-S63 (2005).

61. Parker, G. A. Sexual conflict over mating and fertilization: an overview. Phil. Trans. Roy. Soc. 361, 235-259 (2006).

62. Hosken, D. \& Snook, R. How important is sexual conflict? Am. Nat. 165, S1-S4 (2005).

63. Rowe, L., Cameron, E. \& Day, T. Escalation, retreat and female indifference as alternative outcomes of sexually antagonistic coevolution. Am. Nat. 165, S5-S18 (2005).

64. Hosken, D. J., Stockley, P., Tregenza, T. \& Wedell, N. Monogamy and the battle of the sexes. Ann. Rev. Entomol. 54, 361-378 (2009).

65. Crudgington, H. S., \& Siva-Jothy, M. T. Genital damage, kicking and early death. Nature 407, 855-856 (2000). 
66. Hotzy, C., and Arnqvist, G. Sperm competition favours harmful males in seed beetles. Curr. Biol. 19, 404-407 (2009).

67. Blanckenhorn, W.U., et al. The costs of copulating in the dung fly Sepsis cynipsea. Behav. Ecol. 13, 353-358 (2002).

68. Hosken, D. J. \& Price, T. Genital evolution: the traumas of sex. Curr. Biol. 19, R519-R521 (2009).

69. Rönn, J., Katvala, M., \& Arnqvist, G. Coevolution between harmful male genitalia and female resistance in seed beetles. Proc. Natl. Acad. Sci. USA 104, 1092110925 (2007).

70. Siva-Jothy, M.T. Trauma, disease and collateral damage: conflict in cimicids. Phil. Trans. R. Soc. B 361, 269-275 (2006).

71. Carayon, J. In Monographs of the Cimicidae (ed. Usinger, R.) 81-87 (Entomological Society of America, Philadelphia, 1966).

72. Eberhard, W. G. Sexually antagonistic coevolution in insects is associated with only limited morphological diversity. J. Evol. Biol. 19, 657-681 (2006).

73. Jennions, M. D. \& Kelly, C. D. Geographical variation in male genitalia in Brachyrhaphis episcope (Poeciliidae): is it sexually or naturally selected? Oikos 97, 79-86 (2002).

74. Langerhans, R. B., Layman, C. A. \& DeWitt ,T. J. Male genital size reflects a tradeoff between attracting mates and avoiding predators in two live-bearing fish species. Proc. Natl. Acad. Sci. USA 102, 7618-7623 (2005).

75. Ryan, M.J. A Taste for the Beautiful: The Evolution of Attraction. (Princeton University Press, Princeton, 2018).

76. Wessells, H., Lue, T. F. \& McAninch, J. W. Penile length in the flaccid and erect states: guidelines for penile augmentation. J. Urol. 156, 995-997.

77. Soto, I. M., Manfrin, M. H. \& Hasson, E. Host-dependent phenotypic plasticity of aedeagus morphology in a pair of cactophilic sibling Drosophila species of the replete group (Diptera, Drosophilidae). J. Zool. Syst. Evol. Res. 46, 368-373 (2008).

78. Córdoba-Aguilar, A. Sensory trap as the mechanism of sexual selection in a damselfly genitalic trait (Insecta: Calopterygidae). Am. Nat. 160, 594-601. (2002).

79. Hosken, D .J., Martin, O. Y., Born, J., \& Huber, F. (2003). Sexual conflict in Sepsis cynipsea: female reluctance, fertility and mate choice. J. Evol. Biol. 16, 485-490 (2003). 
80. Morrow, E. H., Arnqvist, G., \& Pitnick, S. (2003). Adaptation versus pleiotropy: why do males harm their mates? Behav. Ecol. 14, 802-806 (2003).

81. Kingsolver, J. G., et. al. The strength of phenotypic selection in natural populations. Am. Nat. 157, 245-261 (2001).

82. Lande, R. \& Arnold, S. J. The measurement of selection on correlated characters. Evolution 37, 1210-1226 (1983).

83. Arnold, S. J. \& Wade, M. J. On the measurement of natural and sexual selection: theory. Evolution 38, 709-719 (1984).

84. Arnold, S. J. \& Wade, M. J. On the measurement of natural and sexual selection: applications. Evolution 38, 720-734 (1984). 


\section{Box 1: Testis size and post-copulatory sexual selection.}

In many animals with internal fertilization, the number of sperm inseminated during copulation is the primary determinant of success during sperm competition ${ }^{38}$. Thus more sperm inseminated frequently means more paternity. Coupled with this, larger testis produce more sperm and hence testis size evolves to match sperm competition risk $^{39-42}$. Stated another way, testis size reflect sperm competition risk, and since sperm competition and cryptic female choice are both associated with females mating with multiple males, testis size reflects female mating patterns and is an accurate measure of the strength of post-copulatory sexual selection (which occurs via sperm competition and cryptic female choice) $)^{38-42}$. 
Table 1 | A comparison of the strength of selection acting on genital and nongenital characters. Shown here are paired $t$-tests of selection gradient estimates in species in which selection on genitals and general morphology were measured in the same animals. Higher selection gradients indicate stronger selection. Gradients are effectively estimated from regression analyses (univariate or multivariate regression) and represent the slope of the associations between a trait (e.g. genital or leg length) and some measure of fitness (e.g. number of offspring or number of mates). So a steep positive gradient would suggest very strong selection for larger traits. Genital evolve relatively quickly and one explanation for this is that they are subjected to especially strong selection. These data tentatively indicate this is not true as the strength of selection acting on genital does not seem strong relative to other traits.

\begin{tabular}{|l|l|l|l|l|c|}
\hline $\begin{array}{l}\text { Selection } \\
\text { gradients }\end{array}$ & $\begin{array}{l}\text { Mean genital } \\
\text { form gradient }\end{array}$ & $\begin{array}{l}\text { Mean general } \\
\text { morphology gradient }\end{array}$ & $t$-statistic & $\begin{array}{l}\text { Degrees } \\
\text { of } \\
\text { freedom }\end{array}$ & $P$-value \\
\hline Univariate & $0.25( \pm 0.10)$ & $0.19( \pm 0.03)$ & 1.025 & 8 & 0.34 \\
\hline Multivariate & $0.14( \pm 0.04)$ & $0.16( \pm 0.04)$ & -0.61 & 7 & 0.56 \\
\hline
\end{tabular}

$t=$ the $t$-test statistic and $P$-value $=$ the significance level of $t$. Data come from Kingsolver et al. ${ }^{5} 81$ which has been updated since the original publication, as well as an ISI Web of Science search using the following search terms: genitalia; genitals; genitalia*selection; genitals*selection; gonopodia; gonopods; pedipalps. The citation histories of the standard references for calculating selection gradients56-58 82-84 were also searched. Studies included are up to 2011. 
Figure 1 | Examples of genital variation. A) A group of closely related flies (Drosophila) that have speciated relatively recently (within the last ca. $2.5 \mathrm{M}$ years) and that are otherwise morphologically very similar (as seen from the images beneath the genital drawings) demonstrate substantial variation in the genital arch (line drawings). B) The cave-dwelling insect Neotrogla curvata is an example of a species in which the female bears the intromittent organ, known as the gynosome, seen in the upper image. The lower image shows how the organ is inserted into the male. C) The greatly elongated penis of the seedbug Lygaeus equestris, which at 7-12 $\mathrm{mm}$ in length, is about threequarters of the length of the male's body. Copyright obtained from 2,4,

Figure 2 | The mechanisms of sexual selection. Sexual selection generally occurs via four mechanisms that generate variation in reproductive success. Male-male competition and female mate choice are the pre-copulatory (classical) mechanisms of sexual selection, and sperm competition and cryptic female choice are the postcopulatory mechanisms. Note that there are sex-role reversed species where females compete more for mates and males are the choosier sex, but generally males are more competitive and females more choosy.

Figure 3 | Evidence for sexual selection acting on male genital form in insects. A) Data showing relative genital dissimilarity (increased distance between points) in two dimensions for two fly genera that either experience postcopulatory sexual selection (polyandrous (P); left plot) or in which postcopulatory sexual selection is relaxed (mongamous (M); right plot). Genitals of polyandrous (P) species are much more diverse than the monogamous (M) species. B) Estimated sexual selection (the shape of the fitness surface) acting on genital form in a beetle. The hill-shaped surface suggests that genital shape is under stabilizing selection (intermediate values are selectively advantaged: have highest fitness returns). The $x$ and $y$ axes show canonical rotations of principal components that describe the shape of the penis (shown as inset figure with the landmarks used to describe its shape). C) The effects of experimental evolution on genital form in the fly Drosophila simulans. The left hand plate shows the shape of the $D$. simulans genital arch before evolution and the right hand images the shape of the $D$. simulans genital arch after evolution with elevated sexual selection, in which females were housed with 4 males for 47 generations (top) and after evolving with relaxed 
sexual selection, in which females were housed with a single male for 47 generations (bottom iamge). Permission obtained from $35,36,54$.

Figure 4 | Schematic representation of the relative size of the penis and testes across the great apes. Relative male body size scaled to a standard-sized female (circles) with penis length (arrows) and testis size (filled spheroids) also shown. Humans have a large penis (pars liberalis length of an erect penis ca. 165mm) compared with our closest relatives, gorillas $(65 \mathrm{~mm})$, orangutans $(85 \mathrm{~mm})$, and chimpanzees $(144 \mathrm{~mm})^{30}$. Chimpanzee have by far the largest testes, reflecting high levels of multiple mating by females and concurrent high levels of sperm competition (Box 1). Gorillas on the other hand have small testes because they are highly monogamous. Humans are somewhere between reflecting a history of moderate sperm competition risk. Note the relative sizes are for illustrative purposes only and are not to scale. Redrawn from ${ }^{21 .}$ 